\title{
Clinical use of the Oncotype DX genomic test to guide treatment decisions for patients with invasive breast cancer
}

This article was published in the following Dove Press journal:

Breast Cancer - Targets and Therapy

29 May 2017

Number of times this article has been viewed

\section{Terri P McVeigh \\ Michael J Kerin}

Discipline of Surgery, Lambe Institute for Translational Research, National University of Ireland Galway, Galway, Republic of Ireland
Correspondence: Terri P McVeigh Discipline of Surgery, Lambe Institute for Translational Research, 2nd Floor, National University of Ireland Galway, Galway, Republic of Ireland

Tel +353 87 791 2374

Email terri.mcveigh@gmail.com

\begin{abstract}
Implementation of the Oncotype DX assay has led to a change in the manner in which chemotherapy is utilized in patients with early stage, estrogen receptor (ER)-positive, node-negative breast cancer; ensuring that patients at highest risk of recurrence are prescribed systemic treatment, while at the same time sparing low-risk patients potential adverse events from therapy unlikely to influence their survival. This test generates a recurrence score between 0 and 100, which correlates with probability of distant disease recurrence. Patients with lowrisk recurrence scores $(0-17)$ are unlikely to derive significant survival benefit with adjuvant chemotherapy and hormonal agents derived from using adjuvant hormonal therapy only. Conversely, adjuvant chemotherapy has been shown to significantly improve survival in patients with high-risk recurrence scores $(\geq 31)$. Trials are ongoing to determine how best to manage patients with recurrence scores in the intermediate range. This review outlines the introduction and impact of Oncotype DX testing on practice; ongoing clinical trials investigating its utility; and challenging clinical scenarios where the absolute recurrence score may require careful interpretation. We also performed a bibliometric analysis of publications on the topics of breast cancer and Oncotype DX as a surrogate marker of acceptability and incorporation of the assay into the management of patients with breast cancer.
\end{abstract}

Keywords: Oncotype DX, gene expression profiling, personalized medicine, precision medicine, breast cancer

\section{Background}

In 2004, the National Surgical Adjuvant Breast and Bowel Project (NSABP) B-14 study showed that the vast majority (78\%) of tamoxifen-treated patients with node-negative estrogen receptor (ER)-expressing tumors will not develop disease relapse within 15 years of diagnosis. ${ }^{1}$ The NSABP B-20 study of the same year showed that the addition of cyclophosphamide, methotrexate, and fluorouracil (CMF) to tamoxifen improved 5-year recurrence-free survival to $89 \% .{ }^{1}$ However, the significant absolute benefit must be balanced with the risk of overtreatment of the significant proportion of women in whom relapse would not have occurred even without the addition of CMF. Traditional prognostic indicators and prognostic scoring systems (e.g., Nottingham Prognostic Index) that have been used to guide decision making with respect to application of adjuvant therapy in breast cancer include patient factors, such as age, and tumor factors, such as grade and stage. ${ }^{2}$ Such tumor factors, particularly size, may reflect a time delay in presentation, as opposed to the biological activity of the disease. ${ }^{3}$ It may be that the tendency of a tumor to metastasize is not simply related to chronology and 
increasing size, but may instead be derived from inherent biological factors, ${ }^{3}$ and indeed tumor size and nodal status are considered independently as risk factors for recurrence. ${ }^{4}$ It has been known for many years that gene expression can be used to more accurately provide prognostic information; and hierarchical clustering of gene expression patterns can discriminate between tumors of distinct molecular subtypes, ${ }^{5}$ each of which is associated with different patterns of metastasis and response to treatment, independent of stage or grade. ${ }^{6}$ Practically speaking, receptor status is used as a surrogate marker of tumor molecular subtype. ${ }^{7}$ ER-negative tumors, in which human epidermal growth factor receptor 2 (HER2) is amplified, have special predilection for early metastasis, whereas ER-positive and HER2-negative tumors have a lesser tendency to metastasize, even at greater tumor size and higher burdens of nodal disease. ${ }^{4}$ It has been known for many years that the low-grade ER-positive group of tumors also responds less often to neoadjuvant chemotherapy than HER2-overexpressing or triple-negative subtypes, again reflecting inherent differences in biology. ${ }^{8-10}$ Traditional prognostic indicators may, therefore, lack sufficient accuracy in identifying those patients who would benefit most from systemic treatment, and similarly may inappropriately assign patients to adjuvant therapy that would be cured by surgery alone.

\section{Development of the Oncotype DX assay}

The Oncotype DX genomic assay (Genomic Health, Inc., Redwood City, CA, USA) is a clinically validated assay that can be used to predict likelihood of recurrence of early stage breast cancer, and can be used therefore, in decision making with respect to systemic therapy. The Oncotype DX-automated algorithm assigns a score to the expression of 16 cancer-related genes multiplied by a factor specific to the group to which each individual gene is allocated, relative to the mean expression of five reference genes to generate an overall recurrence score (RS; Table 1). ${ }^{11,12}$ The Oncotype DX assay is optimized for formalin-fixed paraffin-embedded tumor samples, and all testing is performed centrally in the clinical reference laboratory of Genomic Health, Inc.

The Oncotype DX assay was validated independently in two studies. ${ }^{13,14}$ The prospective NSABP-B14 study confirmed the RS as a continuous predictor of recurrence, independent of patient age and tumor size. ${ }^{13}$ The NSABP B-20 study provided evidence that the RS can also be used to predict those patients with node-negative, ER-positive cancer in whom adjuvant chemotherapy will confer greatest disease-free and overall survival advantage compared to endocrine therapy alone. ${ }^{15}$

Table I Genes included in Oncotype DX assay

\begin{tabular}{|c|c|c|c|}
\hline Gene & Gene function & Scoring group & $\begin{array}{l}\text { Multiplication } \\
\text { factor }\end{array}$ \\
\hline Grb7 & Signaling protein recruited to various tyrosine kinases, including HER2/neu & HER2 & 0.47 \\
\hline HER2 & Growth factor receptor. Overexpression leads to conversion to an oncogene & & 0.47 \\
\hline$E R$ & Membrane receptors which can modify intracellular signaling & Estrogen & -0.34 \\
\hline$P R$ & & & -0.34 \\
\hline$B C L-2$ & Anti-apoptotic oncogene & & -0.34 \\
\hline SCUBE2 & Glycoprotein with a role in $\mathrm{SHH}$ signaling & & -0.34 \\
\hline Ki-67 & Proliferation marker & Proliferation & 1.04 \\
\hline STKI5 & Stabilization of chromosomes during mitotic segregation & & 1.04 \\
\hline Survivin & Inhibition of apoptosis & & 1.04 \\
\hline Cyclin BI & $\begin{array}{l}\text { Component of maturation-promoting factor, stimulation of the } M \text { phase of the } \\
\text { cell cycle }\end{array}$ & & 1.04 \\
\hline MYBL2 & Cell cycle progression & & 1.04 \\
\hline MMPII & Encodes stomelysin 3, important in tissue remodeling & Invasion & 0.1 \\
\hline CTSL2 & Encodes cathepsin L2, stimulates hydrogen peroxide production & & 0.1 \\
\hline CD68 & $\begin{array}{l}\text { Upregulated in breast cancer lines that have a high capacity to metastasize } \\
\text { to bone }\end{array}$ & & 0.05 \\
\hline GSTMI & Glutathione S-transferase, detoxification & & -0.08 \\
\hline BAGI & BCL-2-associated athanogene; enhances the anti-apoptotic effects of BCL-2 & & -0.07 \\
\hline B-actin & Cytoskeletal actin, important for cell motility and structure & Reference genes & 1 \\
\hline GAPDH & Carbohydrate metabolism & & 1 \\
\hline RPLPO & Encodes component of ribosomal 605 subunit & & I \\
\hline GUS & $\beta$-glucuronidase & & 1 \\
\hline TFRC & Transferrin receptor & & \\
\hline
\end{tabular}

Abbreviation: $\mathrm{SHH}$, sonic hedgehog. 
These early validation studies categorized RS as low$(0-17)$, intermediate- $(18-30)$, or high-risk $(\geq 31)$. Paik et al determined Kaplan-Meier estimates of the rate of distant recurrence at 10 years as $6.8 \%, 14.3 \%$, and $30.5 \%$ for patients with low-, intermediate-, and high-risk scores, respectively, and found that the RS was also shown to predict disease recurrence at 10 years when treated as a continuous variable, ${ }^{13}$ more accurately than the adjuvant! online tool. ${ }^{16}$ Habel et al also showed RS to be an independent predictor of breast cancer-related mortality in tamoxifentreated patients with ER-positive, node-negative disease, with 10 -year risk of death $2.8 \%, 10.7 \%$, and $15.5 \%$ in low-, intermediate-, and high-risk patients, respectively. ${ }^{14}$ Other genomic assays exist that purport to have higher predictive value for later onset disease recurrence ${ }^{17-19}$ but their clinical utility in deciding in whom adjuvant chemotherapy will be beneficial above the use of extended endocrine therapy has not been fully elucidated. The beneficial impact of adjuvant systemic chemotherapy is in prevention of early disease recurrence. ${ }^{20,21}$

\section{Clinical implementation}

The increasing incorporation of Oncotype DX into clinical trials or routine clinical treatment of breast cancer can be illustrated by analysis of the number of publications based on these topics. Interrogation of the Web of Science Core Collection (31/10/2016) for publications based on topics "Oncotype
DX" and "Breast cancer" using Boolean operators OR and AND and search terms "oncotype dx OR Oncotype DX OR oncotypedx OR oncotypeDX" AND "breast cancer OR breast carcinoma OR breast malignancy" generated 530 records (Figure 1). The first manuscripts based on these topics were published in 2005, with dramatic increase in publications generated in the years following introduction of Oncotype DX to the market, and with incorporation of the assay into the clinical recommendations of American Society of Clinical Oncology in 2007 and St Gallen international expert consensus conference in 2009, and with increasing numbers in the years of publication of results of key trials such as NSABP B-14 and B-20 in 2010, transATAC in 2013, and TAILORx in 2015. The gradual increase in publications per year may also reflect the lag time in routine access to Oncotype DX in countries in which publicly funded health systems operate, for example, Ireland (2011), England, and Wales (2013).

The use of Oncotype DX RS in determining application of adjuvant chemotherapy has been incorporated into numerous national and international clinical recommendations and guidelines, including those of National Institute of Clinical Excellence, ${ }^{22}$ National Comprehensive Cancer Network $(\mathrm{NCCN}),{ }^{23}$ and European Society for Medical Oncology ${ }^{24}$ and has been successfully implemented in a number of countries, albeit with some regional variation in application, patient selection, and reliance of RS in decision making alongside traditional prognostic indicators. ${ }^{25-27}$

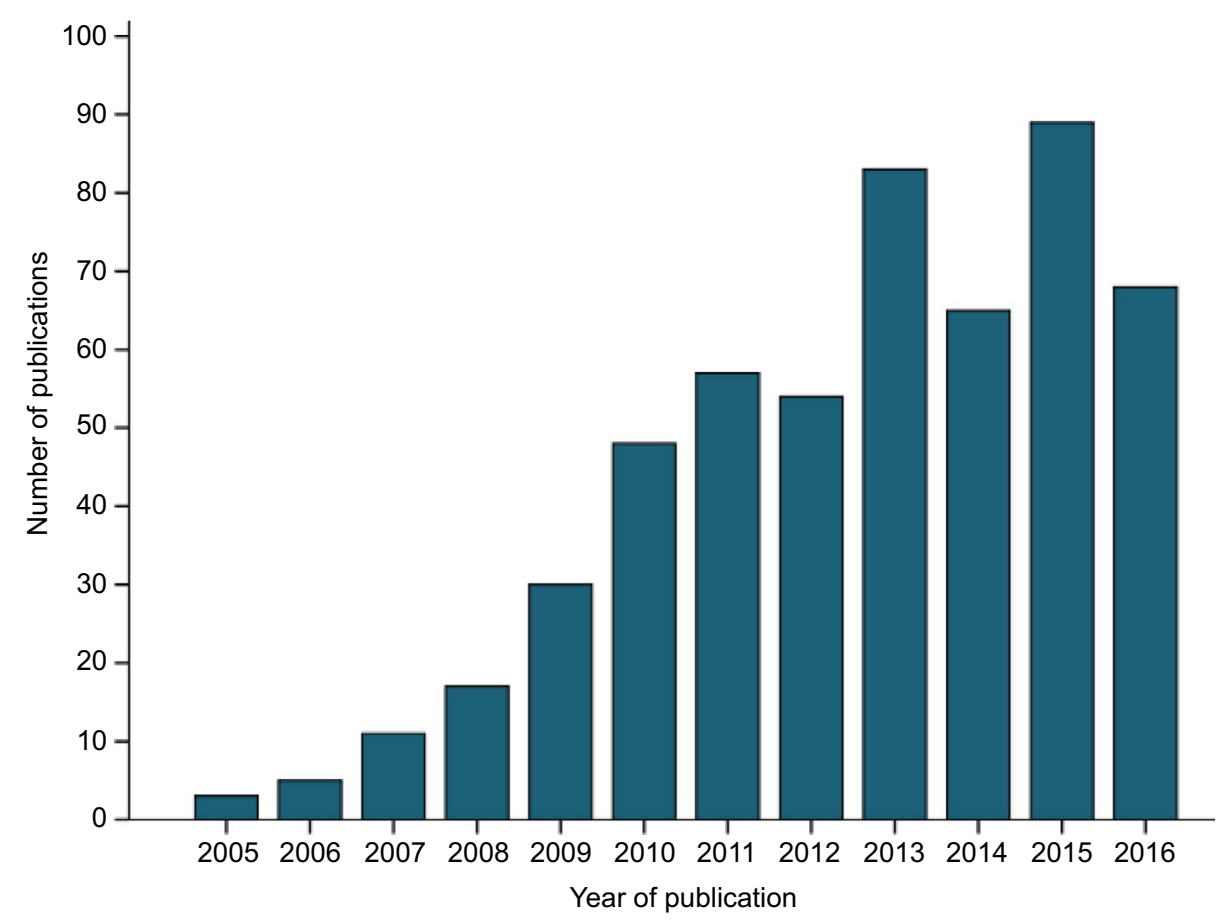

Figure I Publications per annum on the topic related to "Oncotype DX" and "Breast cancer". 
Incorporation of the Oncotype DX assay into routine clinical care has had a significant impact in decision making with respect to chemotherapy prescribing; with certain authors reporting a direct change in management plan in response to the RS in $31 \%$ to $44 \%$ patients, the change in most cases being from a decision to prescribe both chemotherapy and endocrine therapy to an endocrine-only regime. ${ }^{28-32} \mathrm{We}$ have also identified a shift in prescribing patterns among Irish medical oncologists in the years since the development of Oncotype DX, even in intervals during which the assay could not be utilized, ${ }^{26}$ reflecting a change in the paradigm of management of ER-positive lower-risk tumors.

Observations that the gene expression profile of ductal carcinoma in situ (DCIS) associated with invasive disease mirrored that of the invasive component ${ }^{33}$ prompted investigation into the use of the assay in DCIS as a predictor of invasion and recurrence. The Oncotype DX DCIS assay was developed by modifying the 21-gene assay, eliminating analysis of expression of genes related to proliferation. ${ }^{34}$ Description of the use of this assay in DCIS is outside the scope of this article.

\section{TAILORx: Redefining risk categories}

While it is evident from these robust studies that patients with the lowest-risk RS clearly do not derive any additional disease-free or overall survival benefit from the addition of adjuvant chemotherapy to hormonal agents, and conversely, those individuals with highest scores derive substantial benefit from systemic treatment; it is unclear at what absolute score patients in the intermediate-risk category start to derive benefit. It is hoped that results of the TAILORx (Trial Assigning IndividuaLized Options for treatment [Rx]) will help to define the role of chemotherapy in patients with intermediate RS. However, to try to minimize the risk of undertreatment, the authors of this trial redefined the risk categories, with a reduced thresholds for low- $(\mathrm{RS}<11)$, intermediate- (11-25), and high-risk ( $>25)$ categories. ${ }^{35}$ As part of this study, patients with scores $\leq 10$ received hormonal agents only, patients with scores $>25$ received both adjuvant chemotherapy and hormonal therapy, whereas patients in the newly defined intermediate-risk category were randomized to receive either combination chemo- and hormonal therapy or hormonal monotherapy. While the majority of patients in the NSABP-B14 study had scores categorized as low-risk (51\%), and lesser proportions as intermediate-risk (22\%) and high-risk (27\%), ${ }^{13}$ using these new definitions, the vast majority of individuals in the TAILORx study (6907 [67\%]) had scores now considered intermediate-risk, with only
$16.9 \%$ and $15.9 \%$ of patients being considered at high- and low-risk, respectively. ${ }^{20}$

Unsurprisingly, redefinition of the risk categories by TAILORx has created controversy in the management of patients with scores between 11 and 17 . Wen et $\mathrm{al}^{36}$ analyzed the outcome of a large cohort patients with $\mathrm{RS}<18$, specifically analyzing the cohorts with RS 0-10 and 11-17 separately. In keeping with early results of TAILORx, ${ }^{20}$ very low rates of disease recurrence at 5 years was recorded in patients with RS $<11(0.2 \%)$. In this study, only $4 \%$ of patients with RS $0-10$ received chemotherapy, compared to $17 \%$ of patients with RS 11-17. The rate of distant relapse in patients with RS 0-10 was $0.2 \%$, compared to $0.6 \%$ in patients with RS $11-17$. Of patients who relapsed $(n=6)$, only one had an RS $<11$. Only one patient with recurrent disease had received adjuvant chemotherapy, and had an RS of 12. The incidence of disease recurrence in that study was noted to be significantly higher in patients $<40$ years, suggesting that traditional prognostic factors should be considered in patient with intermediate RS.

The interpretation of the data from the intermediate-risk group in TAILORx is awaited, but will hopefully go some way to addressing the lack of clarity that exists surrounding the benefit of chemotherapy in this group. Currently, management of the patient with an intermediate RS poses a significant clinical challenge. In the absence of evidencebased practice regarding incorporation of Oncotype DX RS, we have observed that oncologists revert back to traditional prognostic indicators in decision making for such patients. ${ }^{26}$ The absolute RS is, however, predictive of chemotherapy use when treated as a continuous variable, even in patients in the intermediate-risk group. ${ }^{26,37}$ At present, the clinical utility of this assay is not optimized, as most patients fall into this group, and only a small proportion of patients are ultimately spared chemotherapy on the basis of this result. This not only has clinical drawbacks, but also negates the cost-saving if the test does not alter management.

\section{Utility of Oncotype DX in patients with positive nodal disease}

Most data to date support the use of Oncotype DX profiling of tumors where nodal metastases have not occurred. Standard of care of patients with nodal metastases from ER-positive breast cancer is to offer adjuvant chemotherapy alongside hormonal modulators. ${ }^{23,24}$ However, compared to ERnegative, node-positive disease, where the survival benefit conferred by chemotherapy is unequivocal, several studies have suggested that patients with ER-positive, node-positive disease do not derive as great a survival benefit from systemic 
treatment..$^{38}$ The Southwest Oncology Group (SWOG)-8814 trial provided evidence that chemotherapy in advance of tamoxifen increased disease-free and overall survival in patients with postmenopausal, node-positive, ER-positive disease, ${ }^{39}$ but identified a subset of patients within this cohort who did not benefit from systemic treatment. ${ }^{40}$ The tumors of patients enrolled in this study, where available, were retrospectively assessed using the Oncotype DX assay, and RS were analyzed in the context of patient outcome. These data suggested that the Oncotype DX RS may be used to discriminate between patients with node-positive, ER-positive disease who will benefit from chemotherapy from those who will not, and the RS risk categories could stratify those patients at greatest risk of disease relapse and mortality at 10 years. ${ }^{40}$ The TransATAC ${ }^{41}$ and ECOG2197 $7^{42}$ studies have provided robust evidence that the Oncotype DX RS significantly correlates with recurrence and with time to distant recurrence in both N0 and N+ subgroups. The NSABP-B28 study further showed that RS was an independent predictor of disease-free and overall survival, as well as of recurrence-free interval in postmenopausal women with ER-positive disease managed with chemo- and hormonal therapy. ${ }^{43}$ The Phase III SWOG S1007/RxPONDER trial (NCT01272037) is still ongoing and aims to investigate the role of chemotherapy in patients with 1-3 positive lymph nodes and $\mathrm{RS} \leq 25 .{ }^{44}$ Based on the analysis of data from the SWOG-8814 study, the NCCN guidelines have already been adjusted to include consideration of the assay in patients with 1-3 positive lymph nodes to guide treatment decisions. ${ }^{23}$

\section{Cost-effectiveness}

Despite the high direct cost of the assay and heterogeneity of assay and health care costs between and within countries, many studies from different regions have shown the use of Oncotype DX in patients with node-negative disease to be cost-effective. ${ }^{45-47}$ There is also significant evidence to suggest that it might be cost-effective in patients with node-positive disease ${ }^{31,48,49}$ However, the cost-saving utility of the assay is derived from patients spared chemotherapy. Currently, although many similar assays exist, the Oncotype DX assay is the only such tool that is validated for use in predicting response to chemotherapy; such monopoly as well as patent protection means that the cost of the assay is unlikely to be reduced ${ }^{48}$ Furthermore, if the new risk category definitions as outlined in TAILORx are adopted, fewer patients will fall into the low-risk category. It is possible, therefore, that fewer patients will be spared chemotherapy than initial studies predicted, meaning that projections of costs saved might be overestimated.

\section{Challenging clinical scenarios}

In the case of synchronous bilateral breast cancer, the patient is at risk of relapse of either of the tumors. Where there is discordance in the molecular subtypes of the tumors, the use of Oncotype DX may not be appropriate. Where both tumors are ER-positive and HER2-negative; the utility of the Oncotype DX assay depends on the RS of each tumor. Some authors have found high rates of concurrence in RSs between bilateral tumors, particularly in older patients and in cases of tumors with high levels of progesterone receptor (PgR) expression. ${ }^{50}$

Breast tumors in patients with germline mutations in BRCA1 and BRCA2 demonstrate different biological characteristics than patients with wild-type genotypes, with distinct somatic mutational signatures reflective of deficient homology-directed repair mechanisms. ${ }^{51,52}$ Deficient repair mechanisms may explain why tumors in $B R C A 1 / 2$ gene mutation carriers may be more susceptible to the impact of chemotherapy; and application of chemotherapy in this cohort, even in early stage cancer, has been found to confer significant survival advantage. ${ }^{53,54}$ The majority of breast cancers in patients with $B R C A 1$ gene mutations are triple negative, typically basal subtype. The spectrum of breast cancer phenotypes in BRCA2 gene mutation carriers is much more heterogeneous. ${ }^{55,56}$ Shah et al retrospectively investigated the utility of Oncotype DX in patients with $B R C A 1 / B R C A 2$ gene mutations and ER-positive breast cancer. ${ }^{57}$ Mutation carriers were more likely than age-matched controls to have RS in the intermediate- or high-risk range and overall had higher median RS. This trend has been observed by other authors. ${ }^{58}$ Significantly, cases were also noted to have tumors of higher grade and lower PgR expression scores. Overall, mutation carriers were more likely, therefore, to receive chemotherapy, but proportions receiving chemotherapy within the risk categories were equivalent between carriers and noncarriers. Interestingly, within this study cohort, ER-positive cancers in $B R C A 1$ mutation carriers tended to be of low risk more often than those in BRCA2 mutation carriers. Loss of heterozygosity analyses were not performed on all tumors. Furthermore, this subtype of breast cancer is not usually associated with germline BRCA1 mutations. The possibility that these tumors represent incidental sporadically occurring cancers cannot be dismissed; and in these patients, application of the Oncotype DX assay may be entirely appropriate, as the supposed favorable impact of chemotherapy conferred by BRCA deficiency may not be present if the tumor happened by chance. Further validation of this assay in this particularly unique subgroup of patients is required before implementation can be supported. Furthermore, its use in patients with germline mutations in 
other cancer predisposition genes (e.g., TP53 and STK11) has not yet been investigated.

\section{Patient and clinician attitudes}

Qualitative studies have revealed numerous factors influencing uptake of genomic profiling of tumors among oncologists. Most factors pertain to organizational/logistic and inter- and intrapersonal factors. ${ }^{59}$ Furthermore, uncertainty regarding management of the intermediate-risk cohort represents a significant barrier to uptake. ${ }^{60}$ Other factors that have been identified include concern with respect to patient understanding of the result and implications for treatment ${ }^{61}$ There is a wide variability in reported rates of patient misunderstanding about the risk of recurrence estimation between different centers $(11 \%-83 \%){ }^{62}$ Generally, attitudes of clinicians and patients are positive, and encourage incorporation of this technology into routine use. However, certain subsets of patients, particularly patients with high ${ }^{63}$ or intermediaterisk $^{64} \mathrm{RS}$, experience significant levels of distress and anxiety that cannot be discounted.

\section{Conclusion}

Oncotype DX has led to a shift in the paradigm of treatment of early-stage ER-positive breast cancer, with reduction in overall chemotherapy prescription in low-risk individuals. This shift is reflective of the move toward precision medicine in all realms of oncology in recent times. The application of this assay will spare a large proportion of patients with unnecessary over-treatment, while assuring that those patients with unfavorable biology will receive the whole gamut of available therapies to try to provide most survival benefit as possible. A number of challenges in its application still exist, for example, in patients with tumors that display biological intra-tumor heterogeneity, in patients with germline genetic mutations, or in patients with unfavorable traditional prognostic indicators and apparently conflicting RS categorization; and further longer term follow up is required for these particular patients. We await the results of the TAILORx and RxPONDER trials, which will help inform practice for intermediate-risk patients and patients with low nodal disease burden.

\section{Acknowledgments}

Dr McVeigh was supported by the Health Research Board/ Health Service Executive National Specialist Registrar Academic Fellowship Programme (Grant number NSAFP/14/1), and by Breast Cancer Research (chy9997).

\section{Disclosure}

The authors report no conflicts of interest in this work.

\section{References}

1. Fisher B, Jeong JH, Bryant J, et al; National Surgical Adjuvant Breast and Bowel Project randomised clinical trials. Treatment of lymph-nodenegative, oestrogen-receptor-positive breast cancer: long-term findings from National Surgical Adjuvant Breast and Bowel Project randomised clinical trials. Lancet. 2004;364(9437):858-868.

2. Galea MH, Blamey RW, Elston CE, Ellis IO. The Nottingham Prognostic Index in primary breast cancer. Breast Cancer Res Treat. 1992;22(3):207-219.

3. Oakman C, Bessi S, Zafarana E, Galardi F, Biganzoli L, Di Leo A. Recent advances in systemic therapy: new diagnostics and biological predictors of outcome in early breast cancer. Breast Cancer Res. 2009; 11(2):205

4. Foulkes WD. Size surprise? Tumour size, nodal status, and outcome after breast cancer. Curr Oncol. 2012;19(5):241-243.

5. Sorlie T, Perou CM, Tibshirani R, et al. Gene expression patterns of breast carcinomas distinguish tumor subclasses with clinical implications. Proc Natl Acad Sci U S A. 2001;98(19):10869-10874.

6. Blows FM, Driver KE, Schmidt MK, et al. Subtyping of breast cancer by immunohistochemistry to investigate a relationship between subtype and short and long term survival: a collaborative analysis of data for 10,159 cases from 12 studies. PLoS Med. 2010;7(5):e1000279.

7. Goldhirsch A, Wood WC, Coates AS, Gelber RD, Thürlimann B, Senn HJ; Panel members. Strategies for subtypes-dealing with the diversity of breast cancer: highlights of the St. Gallen International Expert Consensus on the Primary Therapy of Early Breast Cancer 2011. Ann Oncol. 2011;22(8):1736-1747.

8. McVeigh TP, Al-Azawi D, Kearney DE, et al. Assessing the impact of neoadjuvant chemotherapy on the management of the breast and axilla in breast cancer. Clin Breast Cancer. 2014;14(1):20-25.

9. Boughey JC, McCall LM, Ballman KV, et al. Tumor biology correlates with rates of breast-conserving surgery and pathologic complete response after neoadjuvant chemotherapy for breast cancer: findings from the ACOSOG Z1071 (Alliance) Prospective Multicenter Clinical Trial. Ann Surg. 2014;260(4):608-614; discussion 614-616.

10. Lippman ME, Allegra JC, Thompson EB, et al. The relation between estrogen receptors and response rate to cytotoxic chemotherapy in metastatic breast cancer. N Engl J Med. 1978;298(22):1223-1228.

11. Cronin M, Sangli C, Liu ML, et al. Analytical validation of the Oncotype DX genomic diagnostic test for recurrence prognosis and therapeutic response prediction in node-negative, estrogen receptor-positive breast cancer. Clin Chem. 2007;53(6):1084-1091.

12. Sparano JA, Paik S. Development of the 21-gene assay and its application in clinical practice and clinical trials. J Clin Oncol. 2008;26(5):721-728.

13. Paik S, Shak S, Tang G, et al. A multigene assay to predict recurrence of tamoxifen-treated, node-negative breast cancer. $N$ Engl J Med. 2004;351(27):2817-2826.

14. Habel LA, Shak S, Jacobs MK, et al. A population-based study of tumor gene expression and risk of breast cancer death among lymph node-negative patients. Breast Cancer Res. 2006;8(3):R25.

15. Paik S, Tang G, Shak S, et al. Gene expression and benefit of chemotherapy in women with node-negative, estrogen receptor-positive breast cancer. J Clin Oncol. 2006;24(23):3726-3734.

16. Tang G, Shak S, Paik S, et al. Comparison of the prognostic and predictive utilities of the 21-gene Recurrence Score assay and adjuvant! for women with node-negative, ER-positive breast cancer: results from NSABP B-14 and NSABP B-20. Breast Cancer Res Treat. 2011;127(1):133-142.

17. Sgroi DC, Sestak I, Cuzick J, et al. Prediction of late distant recurrence in patients with oestrogen-receptor-positive breast cancer: a prospective comparison of the breast-cancer index (BCI) assay, 21-gene recurrence score, and IHC4 in the TransATAC study population. Lancet Oncol. 2013;14(11):1067-1076.

18. Sestak I, Cuzick J, Dowsett M, et al. Prediction of late distant recurrence after 5 years of endocrine treatment: a combined analysis of patients from the Austrian breast and colorectal cancer study group 8 and arimidex, tamoxifen alone or in combination randomized trials using the PAM50 risk of recurrence score. J Clin Oncol. 2015;33(8):916-922. 
19. Sestak I, Dowsett M, Zabaglo L, et al. Factors predicting late recurrence for estrogen receptor-positive breast cancer. J Natl Cancer Inst. 2013;105(19):1504-1511.

20. Sparano JA, Gray RJ, Makower DF, et al. Prospective validation of a 21-gene expression assay in breast cancer. N Engl J Med. 2015;373(21): 2005-2014.

21. Early Breast Cancer Trialists' Collaborative Group (EBCTCG), Peto R, Davies C, Godwin J, et al. Comparisons between different polychemotherapy regimens for early breast cancer: meta-analyses of long-term outcome among 100,000 women in 123 randomised trials. Lancet. 2012;379(9814):432-444.

22. NICE. Gene expression profiling and expanded immunohistochemistry tests for guiding adjuvant chemotherapy decisions in early breast cancer management: MammaPrint, Oncotype DX, IHC4 and Mammostrat NICE Diagnostics guidance [DG10]; 2013. Available from: https:// www.nice.org.uk/guidance/dg10. Accessed March 1, 2017.

23. NCCN.org. National Comprehensive Cancer Network (NCCN) Clinical Practic Guidelines in Oncology -Breast Cancer (Version 2.2016); 2016. Available from: http:/www.nccn.org/professionals/physicians_gls/pdf/ breast.pdf. Accessed March 1, 2017.

24. Senkus E, Kyriakides S, Ohno S, et al; ESMO Guidelines Committee. Primary breast cancer: ESMO Clinical Practice Guidelines for diagnosis, treatment and follow-up. Ann Oncol. 2015;26 (Suppl 5):v8-v30.

25. Dinan MA, Mi X, Reed SD, Hirsch BR, Lyman GH, Curtis LH. Initial Trends in the use of the 21-gene recurrence score assay for patients with breast cancer in the medicare population, 2005-2009. JAMA Oncol. 2015;1(2):158-166.

26. McVeigh TP, Hughes LM, Miller N, et al. The impact of Oncotype DX testing on breast cancer management and chemotherapy prescribing patterns in a tertiary referral centre. Eur J Cancer. 2014;50(16):2763-2770.

27. Jasem J, Amini A, Rabinovitch R, et al. 21-Gene recurrence score assay as a predictor of adjuvant chemotherapy administration for early-stage breast cancer: an analysis of use, therapeutic implications, and disparity profile. J Clin Oncol. 2016;34(17):1995-2002.

28. Henry LR, Stojadinovic A, Swain SM, Prindiville S, Cordes R, Soballe PW. The influence of a gene expression profile on breast cancer decisions. J Surg Oncol. 2009;99(6):319-323.

29. Lo SS, Mumby PB, Norton J, et al. Prospective multicenter study of the impact of the 21-gene recurrence score assay on medical oncologist and patient adjuvant breast cancer treatment selection. J Clin Oncol. 2010;28(10):1671-1676.

30. Asad J, Jacobson AF, Estabrook A, et al. Does oncotype DX recurrence score affect the management of patients with early-stage breast cancer? Am J Surg. 2008;196(4):527-529.

31. Loncaster J, Armstrong A, Howell S, et al. Impact of oncotype DX breast recurrence score testing on adjuvant chemotherapy use in early breast cancer: real world experience in greater Manchester, UK. Eur J Surg Oncol. Epub 2017 Jan 9.

32. Rutter CE, Yao X, Mancini BR, et al. Influence of a 21-gene recurrence score assay on chemotherapy delivery in breast cancer. Clin Breast Cancer. 2016;16(1):59-62.

33. Baehner, F, Sangli C, Millward C, Cherbavaz D, Goddard A, Shak S. Quantitative gene expression analysis using Oncotype DX in ductal carcinoma in situ that is adjacent to invasive ductal carcinoma. Cancer Res. 2009;69 (Suppl 2):2066.

34. Solin LJ, Gray R, Baehner FL, et al. A multigene expression assay to predict local recurrence risk for ductal carcinoma in situ of the breast. J Natl Cancer Inst. 2013;105(10):701-710.

35. Sparano JA. TAILORx: trial Assigning Individualized Options for Treatment (Rx). Clin Breast Cancer. 2006;7(4):347-350.

36. Wen HY, Krystel-Whittemore M2, Patil S, et al. Breast carcinoma with an Oncotype Dx recurrence score <18: rate of distant metastases in a large series with clinical follow-up. Cancer. 2017;123(1):131-137.

37. Albanell J, Svedman C2, Gligorov J, et al. Pooled analysis of prospective European studies assessing the impact of using the 21-gene recurrence score assay on clinical decision making in women with oestrogen receptor-positive, human epidermal growth factor receptor 2-negative early-stage breast cancer. Eur J Cancer. 2016;66:104-113.
38. Berry DA, Cirrincione C, Henderson IC, et al. Estrogen-receptor status and outcomes of modern chemotherapy for patients with node-positive breast cancer. JAMA. 2006;295(14):1658-1667.

39. Albain KS, Barlow WE, Ravdin PM, et al; Breast Cancer Intergroup of North America. Adjuvant chemotherapy and timing of tamoxifen in postmenopausal patients with endocrine-responsive, node-positive breast cancer: a phase 3, open-label, randomised controlled trial. Lancet. 2009;374(9707):2055-2063.

40. Albain KS, Barlow WE, Shak S, et al; Breast Cancer Intergroup of North America. Prognostic and predictive value of the 21-gene recurrence score assay in postmenopausal women with node-positive, oestrogenreceptor-positive breast cancer on chemotherapy: a retrospective analysis of a randomised trial. Lancet Oncol. 2010;11(1):55-65.

41. Dowsett M, Cuzick J, Wale C, et al. Prediction of risk of distant recurrence using the 21-gene recurrence score in node-negative and node-positive postmenopausal patients with breast cancer treated with anastrozole or tamoxifen: a TransATAC study. J Clin Oncol. 2010;28(11):1829-1834.

42. Goldstein LJ, Gray R, Badve S, et al. Prognostic utility of the 21-gene assay in hormone receptor-positive operable breast cancer compared with classical clinicopathologic features. J Clin Oncol. 2008;26(25): 4063-4071.

43. Mamounas E, Tang G, Paik S, et al. Prognostic impact of the 21-gene recurrence score (RS) on disease-free and overall survival of node-positive, ER-positive breast cancer patients (pts) treated with adjuvant chemotherapy: results from NSABP B-28. J Clin Oncol. 2012;30 (Supp1 27). Abstract 1 .

44. Sun Z, Prat A, Cheang MC, Gelber RD, Perou CM. Chemotherapy benefit for 'ER-positive' breast cancer and contamination of nonluminal subtypes-Waiting for TAILORx and RxPONDER. Ann Oncol. 2015;26(1):70-74.

45. Bargallo-Rocha JE, Lara-Medina F, Pérez-Sánchez V, et al. Cost-effectiveness of the 21-gene breast cancer assay in Mexico. Adv Ther. 2015; 32(3):239-253.

46. Smyth L, Watson G2, Walsh EM, et al. Economic impact of 21-gene recurrence score testing on early-stage breast cancer in Ireland. Breast Cancer Res Treat. 2015;153(3):573-582.

47. Kip M, Monteban H, Steuten L. Long-term cost-effectiveness of Oncotype $\mathrm{DX}^{\mathbb{B}}$ versus current clinical practice from a Dutch cost perspective. J Comp Eff Res. 2015;4(5):433-445.

48. Hall PS, McCabe C, Stein RC, Cameron D. Economic evaluation of genomic test-directed chemotherapy for early-stage lymph node-positive breast cancer. J Natl Cancer Inst. 2012;104(1):56-66.

49. Brufsky AM. Predictive and prognostic value of the 21-gene recurrence score in hormone receptor-positive, node-positive breast cancer. Am J Clin Oncol. 2014;37(4):404-410.

50. Karsten M, Stempel M, Radosa J, Patil S, King TA. Oncotype DX in bilateral synchronous primary invasive breast cancer. Ann Surg Oncol. 2016;23(2):471-476.

51. Alexandrov LB, Nik-Zainal S, Wedge DC, et al. Signatures of mutational processes in human cancer. Nature. 2013;500(7463):415-421.

52. Nik-Zainal S, Davies H1, Staaf J, et al. Landscape of somatic mutations in 560 breast cancer whole-genome sequences. Nature. 2016;534(7605):47-54.

53. Arun B, Bayraktar S, Liu DD, et al. Response to neoadjuvant systemic therapy for breast cancer in BRCA mutation carriers and noncarriers: a single-institution experience. J Clin Oncol. 2011;29(28):3739-3746.

54. Narod SA, Metcalfe K, Lynch HT, et al. Should all BRCA1 mutation carriers with stage I breast cancer receive chemotherapy? Breast Cancer Res Treat. 2013;138(1):273-279.

55. Foulkes WD, Stefansson IM, Chappuis PO, et al. Germline BRCA1 mutations and a basal epithelial phenotype in breast cancer. $J$ Natl Cancer Inst. 2003;95(19):1482-1485.

56. Lakhani SR, Van De Vijver MJ, Jacquemier J, et al. The pathology of familial breast cancer: predictive value of immunohistochemical markers estrogen receptor, progesterone receptor, HER-2, and p53 in patients with mutations in BRCA1 and BRCA2. J Clin Oncol. 2002;20(9): 2310-2318. 
57. Shah PD, Patil S, Dickler MN, Offit K, Hudis CA, Robson ME. Twentyone-gene recurrence score assay in BRCA-associated versus sporadic breast cancers: differences based on germline mutation status. Cancer. 2016;122(8):1178-1184.

58. Lewin R, Sulkes A, Shochat T, et al. Oncotype-DX recurrence score distribution in breast cancer patients with BRCA1/2 mutations. Breast Cancer Res Treat. 2016;157(3):511-516.

59. Weldon CB, Trosman JR, Gradishar WJ, Benson AB 3rd, Schink JC. Barriers to the use of personalized medicine in breast cancer. J Oncol Pract. 2012;8(4):e24-e31.

60. Roberts MC, Bryson A, Weinberger M, et al. Oncologists' barriers and facilitators for Oncotype Dx use: qualitative study. Int JTechnol Assess Health Care. 2016;32(5):355-361.
61. Bombard Y, Rozmovits L, Trudeau M, Leighl NB, Deal K, Marshall DA. The value of personalizing medicine: medical oncologists' views on gene expression profiling in breast cancer treatment. Oncologist. 2015; 20(4):351-356.

62. Leggett LE, Lorenzetti DL, Noseworthy T, Tiwana S, Mackean G, Clement F. Experiences and attitudes toward risk of recurrence testing in women with breast cancer: a systematic review. Breast Cancer Res Treat. 2014;144(3):457-465.

63. Tzeng JP, Mayer D, Richman AR, et al. Women's experiences with genomic testing for breast cancer recurrence risk. Cancer. 2010;116(8):1992-2000.

64. O'Neill SC, Brewer NT, Lillie SE, et al. Women's interest in gene expression analysis for breast cancer recurrence risk. J Clin Oncol. 2007;25(29):4628-4634.

\section{Publish your work in this journal}

Breast Cancer - Targets and Therapy is an international, peerreviewed open access journal focusing on breast cancer research, identification of therapeutic targets and the optimal use of preventative and integrated treatment interventions to achieve improved outcomes, enhanced survival and quality of life for the cancer patient.

The manuscript management system is completely online and includes a very quick and fair peer-review system, which is all easy to use. Visit http://www.dovepress.com/testimonials.php to read real quotes from published authors. 